\title{
DISCRETE ANISOTROPIC CURVATURE FLOW OF GRAPHS
}

\author{
Klaus Deckelnick ${ }^{1}$ And Gerhard DziUK ${ }^{1}$
}

\begin{abstract}
The evolution of $n$-dimensional graphs under a weighted curvature flow is approximated by linear finite elements. We obtain optimal error bounds for the normals and the normal velocities of the surfaces in natural norms. Furthermore we prove a global existence result for the continuous problem and present some examples of computed surfaces.
\end{abstract}

Résumé. L'évolution de graphes $n$-dimensionnels selon le problème de flot à courbure pondérée est approchée par des éléments finis linéaires. On obtient des bornes d'erreurs optimales pour les normales et pour les vitesses normales des surfaces, dans des normes naturelles. De plus, nous établissons un théorème d'existence globale pour le problème continu et nous présentons quelques exemples numériques d'évolution de surfaces.

AMS Subject Classification. 65N30, 35K65.

Received: January 9, 1997. Revised: November 21, 1997.

\section{INTRODUCTION}

In phase transition problems one is frequently interested in evolution laws of the form

$$
V=-\frac{1}{\beta(\nu)}\left(\sum_{i=1}^{n+1} \frac{\partial}{\partial x_{i}}\left(\gamma_{p_{i}}(\nu)\right)+c\right) \quad \text { on } \Gamma_{t} .
$$

Here, $\Gamma_{t}$ is a hypersurface in $\mathbb{R}^{n+1}, \nu$ denotes the unit normal to $\Gamma_{t}$ and $V$ is the normal velocity of $\Gamma_{t}$. The function $\gamma: \mathbb{R}^{n+1} \backslash\{0\} \rightarrow \mathbb{R}, \gamma=\gamma(p)$, is positively homogeneous of degree one, i.e.

$$
\gamma(\lambda p)=\lambda \gamma(p), \quad p \in \mathbb{R}^{n+1}, \lambda>0 .
$$

Furthermore, $\gamma_{p_{i}}$ is the derivative of $\gamma$ with respect to $p_{i}$. Finally, $\beta: S^{n} \rightarrow \mathbb{R}$ is positive and continuous and $c$ is a constant.

The law (1.1) arises in the mathematical modeling of the evolution of an interface $\Gamma_{t}$ separating a liquid and a solid phase under the assumption that the free energy in either phase is constant. The constant $c$ in (1.1) then represents the energy difference in the bulk phases while the restriction of $\gamma$ to $S^{n}$ is the surface tension. The function $\beta$ is usually referred to as the kinetic coefficient. For a detailed derivation of (1.1) from the force balances and the second law of thermodynamics see [1].

Keywords and phrases. Mean curvature flow, anisotropic, finite elements, convergence.

1 Institut für Angewandte Mathematik, Hermann-Herder-Straße 10, 79104 Freiburg i. Br., Germany.

e-mail: gerd@mathematik.uni-freiburg.de

(c) EDP Sciences, SMAI 1999 
An important special case occurs for the choices $\gamma(p)=|p|, \beta \equiv 1, c=0$, which reduces (1.1) to the wellknown mean curvature flow $V=-H$. However, in many applications the function $\gamma$ is anisotropic and then the interfaces evolve according to the weighted curvature flow (1.1).

We shall restrict our attention to surfaces $\Gamma_{t}$ which can be represented as the graph of a scalar function $u(x, t)$ over a fixed domain $\Omega \subset \mathbb{R}^{n}$, i.e.

$$
\Gamma_{t}=\{(x, u(x, t)) \mid x \in \Omega\}
$$

In order to keep the presentation simpler we shall henceforth assume that $\beta \equiv 1$ and $c=0$. The unit normal vector $\nu$ to $\Gamma_{t}$ is given by

$$
\nu=\frac{(\nabla u,-1)}{\sqrt{1+|\nabla u|^{2}}}
$$

while the normal velocity is computed as

$$
V=\left(0, u_{t}\right) \cdot \nu=-\frac{u_{t}}{\sqrt{1+|\nabla u|^{2}}} .
$$

Inserting these identities into (1.1) and observing that $\gamma_{p_{i}}\left((\nabla u,-1) / \sqrt{1+|\nabla u|^{2}}\right)=\gamma_{p_{i}}((\nabla u,-1))$ (see (1.4) below) we obtain the following initial boundary value problem

$$
\begin{aligned}
u_{t}-\sqrt{1+|\nabla u|^{2}} \sum_{i=1}^{n} \frac{\partial}{\partial x_{i}}\left(\gamma_{p_{i}}(\nabla u,-1)\right) & =0 & & \text { in } \Omega \times(0, T) \\
u & =u_{0} & & \text { on } \partial \Omega \times(0, T) \\
u(\cdot, 0) & =u_{0} & & \text { in } \Omega .
\end{aligned}
$$

Condition $(1.2)_{2}$ says that the boundary of $\Gamma_{t}$ remains fixed during the evolution, since $u_{0}$ is independent of $t$.

Furthermore, in what follows we assume that $\gamma \in C^{3+\alpha}\left(\mathbb{R}^{n+1} \backslash\{0\}\right)$ for some $\alpha \in(0,1), \gamma(p)>0$ for $p \in \mathbb{R}^{n+1} \backslash\{0\}$ and that there is $\gamma_{0}>0$ such that

$$
\begin{array}{rlrl}
\gamma(\lambda p) & =|\lambda| \gamma(p), & & p \neq 0, \lambda \neq 0 \\
\left\langle D^{2} \gamma(p) q, q\right\rangle \geq \gamma_{0}|q|^{2}, & & |p|=1,\langle q, p\rangle=0 .
\end{array}
$$

Here, $|\cdot|$ and $\langle\cdot, \cdot\rangle$ denote the Euclidean norm and scalar product respectively. It is easy to see that $(1.3)_{1}$ implies the relations

$$
\begin{aligned}
\langle\nabla \gamma(p), p\rangle & =\gamma(p), & \left\langle D^{2} \gamma(p) p, q\right\rangle & =0 \\
\gamma_{p_{i}}(\lambda p) & =\frac{\lambda}{|\lambda|} \gamma_{p_{i}}(p), & \gamma_{p_{i} p_{j}}(\lambda p) & =\frac{1}{|\lambda|} \gamma_{p_{i} p_{j}}(p)
\end{aligned}
$$

for all $p \in \mathbb{R}^{n+1} \backslash\{0\}, q \in \mathbb{R}^{n+1}$ and $\lambda \neq 0$ and $i, j \in\{1, \ldots, n+1\}$.

An existence and uniqueness result for (1.2) will be given in section 4 and essentially follows from the work of Lieberman [11]. For a semidiscrete approximation of (1.2) by linear finite elements we shall prove the error estimate

$$
\int_{0}^{T}\left\|V-V_{h}\right\|_{L^{2}\left(\Gamma_{h, t}\right)}^{2} \mathrm{~d} t+\sup _{t \in(0, T)}\left\|\left(\nu-\nu_{h}\right)(\cdot, t)\right\|_{L^{2}\left(\Gamma_{h, t}\right)}^{2} \leq C h^{2}
$$


where $\nu_{h}$ and $V_{h}$ are appropriate approximations of the normal $\nu$ and the normal velocity $V$, cf. Theorem 2.1 below. This result holds provided the solution $u$ satisfies

$$
\sup _{t \in(0, T)}\left(\|u(\cdot, t)\|_{H^{2, \infty}(\Omega)}+\left\|u_{t}(\cdot, t)\right\|_{H^{1, \infty}(\Omega)}\right)+\int_{0}^{T}\left\|u_{t}\right\|_{H^{2}(\Omega)}^{2}<\infty
$$

which will be guaranteed by the existence theorem. Here, $H^{m, p}(\Omega)$ denotes the usual Sobolev space. The corresponding norm is given by

$$
\|u\|_{H^{m, p}(\Omega)}=\left(\sum_{k=0}^{m}\left\|D^{k} u\right\|_{L^{p}(\Omega)}^{p}\right)^{\frac{1}{p}}
$$

with the usual modification for $p=\infty$. Furthermore we set $H^{m}(\Omega)=H^{m, 2}(\Omega)$ and $H_{0}^{m}(\Omega)$ denotes the closure of $C_{0}^{\infty}(\Omega)$ with respect to $\|\cdot\|_{H^{m}(\Omega)}$.

Existence of a global smooth solution for the (isotropic) mean curvature flow on convex domains also follows from [11] while it was proved for Neumann boundary conditions by Huisken in [8]. Giga [6] examines the evolution of a one-dimensional graph in case $(1.3)_{2}$ is not satisfied. [2] studies anisotropic motion by mean curvature in the context of Finsler geometry and [14] gives a survey of various mathematical approaches to (1.1).

\section{Finite ELEMENT Method}

Our finite element method is based on the following weak formulation of $(1.2)$ : find $u(\cdot, t) \in H^{1, \infty}(\Omega)$ such that $u(\cdot, t)-u_{0}=0$ on $\partial \Omega$ and

$$
\begin{aligned}
\int_{\Omega} \frac{u_{t} \varphi}{\sqrt{1+|\nabla u|^{2}}+\sum_{i=1}^{n} \int_{\Omega} \gamma_{p_{i}}(\nabla u,-1) \varphi_{x_{i}}}=0 \quad \forall \varphi \in H_{0}^{1}(\Omega), t \in(0, T) \\
u(\cdot, 0)=u_{0} \quad \text { in } \Omega .
\end{aligned}
$$

Let us denote by $\mathcal{T}_{h}$ a triangulation of $\Omega$ with maximum mesh size $h:=\max _{S \in \mathcal{T}_{h}} \operatorname{diam}(S)$ and by $\Omega_{h}$ the corresponding discrete domain, i.e.

$$
\Omega_{h}=\bigcup_{S \in \mathcal{T}_{h}} S
$$

We shall assume that all vertices on $\partial \Omega_{h}$ also lie on $\partial \Omega$ and that the triangulation is nondegenerate in the sense that

$$
\max _{S \in \mathcal{T}_{h}} \frac{\operatorname{diam}(S)}{\rho_{S}} \leq \kappa_{0}
$$

where the constant $\kappa_{0}$ is independent of $h$ and $\rho_{S}$ denotes the radius of the largest ball which is contained in $\bar{S}$. We define the finite dimensional space $X_{h}$ by

$$
X_{h}:=\left\{\varphi_{h} \in C^{0}\left(\bar{\Omega}_{h}\right) \mid \varphi_{h} \text { is affine linear on each } S \in \mathcal{T}_{h}\right\}
$$

and $\stackrel{\circ}{X}_{h}:=X_{h} \cap H_{0}^{1}\left(\Omega_{h}\right)$.

We now consider a semidiscrete approximation of $(1.2)$ : find $u_{h}(\cdot, t) \in X_{h}$ with $u_{h}(\cdot, t)-u_{h}^{0} \in \stackrel{\circ}{X}_{h}$ such that

$$
\begin{aligned}
\int_{\Omega_{h}} \frac{u_{h, t} \varphi_{h}}{\sqrt{1+\left|\nabla u_{h}\right|^{2}}}+\sum_{i=1}^{n} \int_{\Omega_{h}} \gamma_{p_{i}}\left(\nabla u_{h},-1\right) \varphi_{h, x_{i}} & =0 \quad \forall \varphi_{h} \in \dot{X}_{h}, t \in[0, T] \\
u_{h}(\cdot, 0) & =u_{h}^{0},
\end{aligned}
$$


where $u_{h}^{0}=I_{h} u_{0} \in X_{h}$ is an interpolant of $u_{0}(c f .(3.2))$.

Our main result gives an error bound for the important quantities $V$ and $\nu$.

Theorem 2.1. Let $u$ be the solution of (1.2) given by Theorem 4.1. Then (2.3) has a unique solution $u_{h}$ and

$$
\int_{0}^{T}\left\|V-V_{h}\right\|_{L^{2}\left(\Gamma_{h, t}\right)}^{2} \mathrm{~d} t+\sup _{t \in(0, T)}\left\|\left(\nu-\nu_{h}\right)(\cdot, t)\right\|_{L^{2}\left(\Gamma_{h, t}\right)}^{2} \leq C h^{2} .
$$

Here, $\Gamma_{h, t}=\left\{\left(x, u_{h}(x, t)\right) \mid x \in \Omega_{h} \cap \Omega\right\}, \nu_{h}=\left(\nabla u_{h},-1\right) / \sqrt{1+\left|\nabla u_{h}\right|^{2}}$,

$$
V(x, t)=-\frac{u_{t}(x, t)}{\sqrt{1+|\nabla u(x, t)|^{2}}}, V_{h}(x, t)=-\frac{u_{h, t}(x, t)}{\sqrt{1+\left|\nabla u_{h}(x, t)\right|^{2}}}
$$

and for $f: \Omega_{h} \rightarrow \mathbb{R}$

$$
\|f\|_{L^{2}\left(\Gamma_{h, t}\right)}^{2}=\int_{\Omega_{h} \cap \Omega} f^{2} \sqrt{1+\left|\nabla u_{h}(\cdot, t)\right|^{2}} .
$$

We emphasize that the error estimate (2.4) is valid for arbitrary space dimension $n$. Note also that the error measured in $L^{2}\left(\Gamma_{h, t}\right)$ dominates the error measured in $L^{2}\left(\Omega_{h} \cap \Omega\right)$. It is possible to include a right hand side in the error analysis provided the corresponding continuous problem has a smooth solution.

The main problem in the convergence analysis arises from the presence of $\sqrt{1+|\nabla u|^{2}}$ in the denominator of $\int_{\Omega}\left(u_{t} \varphi / \sqrt{1+|\nabla u|^{2}}\right)$. This introduces an additional nonlinearity to the one already present in the elliptic part and makes the application of the usual energy arguments difficult. We shall solve this problem by working with the geometric quantities $\nu$ and $V$ as often as possible rather than with $u$ and $\nabla u$.

The proof of Theorem 2.1 will be given in Section 3. As already mentioned above Section 4 contains an existence and uniqueness result for (1.2) and in Section 5 we present numerical examples.

Let us finally mention other work which is related to ours. In [4] the authors proved convergence for a finite element method solving the isotropic mean curvature flow problem with linear finite elements. The proof used differentiation with respect to time of the weak equations together with a deformation technique and so became fairly involved. The result of Theorem 2.1 will contain the isotropic flow and will provide a much simpler proof also for that case. In [7] the surface energy $\gamma$ is approximated by a crystalline one and a convergence analysis for the resulting scheme is given in the case of a graph of one variable. Dobrowolski [5] considers a class of nonlinear parabolic problems with elliptic part in divergence form and proves $L^{\infty}$-convergence for a linear finite element method.

Remark 2.2. The $\gamma$-mean curvature is given by

$$
H_{\gamma}=\sum_{i=1}^{n} \frac{\partial}{\partial x_{i}}\left(\gamma_{p_{i}}(\nabla u,-1)\right)
$$

so that $H_{\gamma}=-V$ according to (1.2). Let us assume that $\Omega_{h}$ is contained in $\Omega$. The discrete $\gamma$-mean curvature $H_{\gamma h}$ (for a piecewise linear surface) first can only be defined as a functional, namely

$$
H_{\gamma h}(\varphi)=-\int_{\Omega_{h}} \sum_{i=1}^{n} \gamma_{p_{i}}\left(\nabla u_{h},-1\right) \varphi_{x_{i}}, \varphi \in H_{0}^{1}\left(\Omega_{h}\right) .
$$

If we define $P_{h} H_{\gamma h}$ to be the $L^{2}$-projection of the discrete curvature $H_{\gamma h}$ onto $\stackrel{\circ}{X}_{h}$,

$$
\left(P_{h} H_{\gamma h}, \varphi_{h}\right)_{L^{2}\left(\Omega_{h}\right)}=H_{\gamma h}\left(\varphi_{h}\right) \forall \varphi_{h} \in \stackrel{\circ}{X}_{h}
$$


then (2.4) also implies an error bound for the $\gamma$-mean curvature

$$
\int_{0}^{T}\left\|H_{\gamma}-P_{h} H_{\gamma h}\right\|_{L^{2}\left(\Omega_{h}\right)}^{2} \mathrm{~d} t \leq C h^{2} .
$$

Note that projecting onto the space with zero boundary values is correct since the differential equation implies that $H_{\gamma}=0$ on $\partial \Omega$. Using the differential equation we obtain

$$
\begin{aligned}
\left(P_{h} H_{\gamma}-P_{h} H_{\gamma h}, \varphi_{h}\right)_{L^{2}\left(\Omega_{h}\right)} & =\left(H_{\gamma}, \varphi_{h}\right)_{L^{2}\left(\Omega_{h}\right)}-H_{\gamma h}\left(\varphi_{h}\right) \\
& =-\left(V-V_{h}, \varphi_{h}\right)_{L^{2}\left(\Omega_{h}\right)}
\end{aligned}
$$

for every $\varphi_{h} \in \stackrel{\circ}{X}_{h}$ and thus

$$
\left\|P_{h} H_{\gamma}-P_{h} H_{\gamma h}\right\|_{L^{2}\left(\Omega_{h}\right)} \leq\left\|V-V_{h}\right\|_{L^{2}\left(\Omega_{h}\right)} .
$$

\section{Proof of Theorem 2.1}

Since $\stackrel{\circ}{X}_{h}$ is finite dimensional, existence and uniqueness of $u_{h}$ on some interval $\left[0, T_{*}\right)$ follow from the theory of ODEs. To see that $u_{h}$ can be continued to $[0, T]$ we prove a bound on $u_{h}$. Since $u_{h, t} \in \dot{X}_{h}$ we obtain from $(2.3)$

$$
\int_{\Omega_{h}} \frac{\left(u_{h, t}\right)^{2}}{\sqrt{1+\left|\nabla u_{h}\right|^{2}}}+\frac{\mathrm{d}}{\mathrm{d} t} \int_{\Omega_{h}} \gamma\left(\nabla u_{h},-1\right)=0, t \in\left(0, T_{*}\right),
$$

so that integration of (3.1) with respect to time implies

$$
\sup _{t \in\left(0, T_{*}\right)} \int_{\Omega_{h}} \gamma\left(\nabla u_{h}(\cdot, t),-1\right) \leq C .
$$

From (1.3) we deduce

$$
\gamma\left(\nabla u_{h},-1\right)=\gamma\left(\frac{\left(\nabla u_{h},-1\right)}{\sqrt{1+\left|\nabla u_{h}\right|^{2}}}\right) \sqrt{1+\left|\nabla u_{h}\right|^{2}} \geq \inf _{|p|=1} \gamma(p)\left|\nabla u_{h}\right|
$$

and therefore

$$
\int_{0}^{T_{*}} \int_{\Omega_{h}} \frac{\left(u_{h, t}\right)^{2}}{\sqrt{1+\left|\nabla u_{h}\right|^{2}}}+\sup _{t \in\left(0, T_{*}\right)}\left\|\nabla u_{h}(\cdot, t)\right\|_{L^{1}\left(\Omega_{h}\right)} \leq C
$$

where $C$ is independent of $T_{*}$. This allows us to extend $u_{h}$ to $[0, T]$.

Our assumptions on the triangulation $\mathcal{T}_{h}$ imply that $\Omega_{h} \subset B_{\delta}(\Omega)=: \Omega^{\prime}$ for small $h$ and some $\delta>0$. Using the results in [3] with minor modifications at the boundary $\partial \Omega$ it is possible to construct for every $v \in H^{2}\left(\Omega^{\prime}\right) \cap H_{0}^{1}(\Omega)$ an element $I_{h} v \in \stackrel{\circ}{X}_{h}$ such that

$$
\left\|v-I_{h} v\right\|_{H^{k}\left(\Omega_{h}\right)} \leq C h\|v\|_{H^{k+1}\left(\Omega_{h}\right)}, \quad k=0,1 .
$$


In view of the regularity of $u$ and $\partial \Omega\left(c f\right.$. Theorem 4.1) there is a function $\bar{u}: \Omega^{\prime} \times(0, T) \rightarrow \mathbb{R}$ such that $\bar{u}_{\mid \Omega \times(0, T)}=u$ and

$$
\begin{aligned}
\|\bar{u}(\cdot, t)\|_{H^{2, \infty}\left(\Omega^{\prime}\right)} & \leq C\|u(\cdot, t)\|_{H^{2, \infty}(\Omega)} \\
\left\|\bar{u}_{t}(\cdot, t)\right\|_{H^{2}\left(\Omega^{\prime}\right)} & \leq C\left\|u_{t}(\cdot, t)\right\|_{H^{2}(\Omega)} \\
\left\|\bar{u}_{t}(\cdot, t)\right\|_{H^{1, \infty}\left(\Omega^{\prime}\right)} & \leq C\left\|u_{t}(\cdot, t)\right\|_{H^{1, \infty}(\Omega)}
\end{aligned}
$$

for a.a. $t \in(0, T)$.

Lemma 3.1. There exists a constant $C>0$ such that

$$
\frac{1}{2} \int_{\Omega_{h}} \frac{\left|\bar{u}_{t}-u_{h, t}\right|^{2}}{\sqrt{1+\left|\nabla u_{h}\right|^{2}}}+\frac{\mathrm{d}}{\mathrm{d} t} E(t) \leq C\left(1+\left\|\bar{u}_{t}\right\|_{H^{2}\left(\Omega_{h}\right)}^{2}\right) h^{2}+C \int_{\Omega_{h}}\left|\bar{\nu}-\nu_{h}\right|^{2} \sqrt{1+\left|\nabla u_{h}\right|^{2}}
$$

where $\bar{\nu}=(\nabla \bar{u},-1) / \sqrt{1+|\nabla \bar{u}|^{2}}$ and

$$
E(t)=\int_{\Omega_{h}}\left[\gamma\left(\nabla u_{h}(\cdot, t),-1\right)-\sum_{i=1}^{n} \gamma_{p_{i}}(\nabla \bar{u}(\cdot, t),-1) u_{h, x_{i}}(\cdot, t)+\gamma_{p_{n+1}}(\nabla \bar{u}(\cdot, t),-1)\right] .
$$

Proof. Using (2.3), integration by parts and (1.2) we obtain for arbitrary $\varphi_{h} \in \stackrel{\circ}{X}_{h}$

$$
\begin{aligned}
& \int_{\Omega_{h}} \frac{\left(\bar{u}_{t}-u_{h, t}\right) \varphi_{h}}{\sqrt{1+\left|\nabla u_{h}\right|^{2}}+\sum_{i=1}^{n} \int_{\Omega_{h}}\left(\gamma_{p_{i}}(\bar{\nu})-\gamma_{p_{i}}\left(\nu_{h}\right)\right) \varphi_{h, x_{i}}=} \int_{\Omega_{h}} \frac{\bar{u}_{t} \varphi_{h}}{\sqrt{1+|\nabla \bar{u}|^{2}}}+\int_{\Omega_{h}} \bar{u}_{t}\left(\frac{1}{\sqrt{1+\left|\nabla u_{h}\right|^{2}}}-\frac{1}{\sqrt{1+|\nabla \bar{u}|^{2}}}\right) \varphi_{h} \\
&-\sum_{i, j=1}^{n} \int_{\Omega_{h}} \gamma_{p_{i} p_{j}}(\nabla \bar{u},-1) \bar{u}_{x_{i} x_{j}} \varphi_{h} \\
&=\int_{\Omega_{h}} \bar{u}_{t}\left(\bar{\nu}_{n+1}-\nu_{h, n+1}\right) \varphi_{h}+\int_{\Omega_{h} \backslash \Omega} d(\bar{u}) \varphi_{h}
\end{aligned}
$$

where

$$
d(\bar{u})=\frac{\bar{u}_{t}}{\sqrt{1+|\nabla \bar{u}|^{2}}}-\sum_{i, j=1}^{n} \gamma_{p_{i} p_{j}}(\nabla \bar{u},-1) \bar{u}_{x_{i} x_{j}} .
$$


Since $u_{t}(\cdot, t)=0$ on $\partial \Omega, u_{h, t}(\cdot, t)=0$ on $\partial \Omega_{h}(3.2)$ implies that $\varphi_{h}:=I_{h} \bar{u}_{t}(\cdot, t)-u_{h, t}(\cdot, t) \in \dot{X}_{h}$ and therefore by (3.4)

$$
\begin{aligned}
& \int_{\Omega_{h}} \frac{\left(\bar{u}_{t}-u_{h, t}\right)^{2}}{\sqrt{1+\left|\nabla u_{h}\right|^{2}}}+\sum_{i=1}^{n} \int_{\Omega_{h}}\left(\gamma_{p_{i}}(\bar{\nu})-\gamma_{p_{i}}\left(\nu_{h}\right)\right)\left(\bar{u}_{t, x_{i}}-u_{h t, x_{i}}\right)=\int_{\Omega_{h}} \frac{\left(\bar{u}_{t}-u_{h, t}\right)\left(\bar{u}_{t}-I_{h} \bar{u}_{t}\right)}{\sqrt{1+\left|\nabla u_{h}\right|^{2}}} \\
&+\sum_{i=1}^{n} \int_{\Omega_{h}}\left(\gamma_{p_{i}}(\bar{\nu})-\gamma_{p_{i}}\left(\nu_{h}\right)\right)\left(\bar{u}_{t, x_{i}}-\left(I_{h} \bar{u}_{t}\right)_{x_{i}}\right)+\int_{\Omega_{h}} \bar{u}_{t}\left(\bar{\nu}_{n+1}-\nu_{h, n+1}\right)\left(I_{h} \bar{u}_{t}-u_{h, t}\right)+\int_{\Omega_{h} \backslash \Omega} d(\bar{u})\left(I_{h} \bar{u}_{t}-u_{h, t}\right) \\
& \equiv \sum_{k=1}^{4} I_{k} .
\end{aligned}
$$

The integrals on the right-hand side are estimated with (3.2) and Young's inequality.

$$
\left|I_{1}\right| \leq\left(\int_{\Omega_{h}} \frac{\left(\bar{u}_{t}-u_{h, t}\right)^{2}}{\sqrt{1+\left|\nabla u_{h}\right|^{2}}}\right)^{\frac{1}{2}}\left\|\bar{u}_{t}-I_{h} \bar{u}_{t}\right\|_{L^{2}\left(\Omega_{h}\right)} \leq \varepsilon \int_{\Omega_{h}} \frac{\left(\bar{u}_{t}-u_{h, t}\right)^{2}}{\sqrt{1+\left|\nabla u_{h}\right|^{2}}}+C_{\varepsilon} h^{2}\left\|\bar{u}_{t}\right\|_{H^{1}\left(\Omega_{h}\right)}^{2} .
$$

Next, we have for $s \in[0,1]$

$$
\begin{aligned}
\left|s \bar{\nu}+(1-s) \nu_{h}\right|^{2} & =s^{2}+(1-s)^{2}+2 s(1-s) \frac{\nabla \bar{u} \cdot \nabla u_{h}+1}{\sqrt{1+|\nabla \bar{u}|^{2}} \sqrt{1+\left|\nabla u_{h}\right|^{2}}} \\
& \geq 1-2 s(1-s)\left(1+\frac{|\nabla \bar{u}|}{\sqrt{1+|\nabla \bar{u}|^{2}}}\right) \geq 1-\frac{1}{2}\left(\frac{M}{\sqrt{1+M^{2}}}+1\right)=: \alpha^{2}>0
\end{aligned}
$$

where $M$ is an upper bound on $\|\nabla \bar{u}\|_{L^{\infty}\left(\bar{\Omega}^{\prime} \times[0, T]\right)}$. The mean value inequality then gives

$$
\left|I_{2}\right| \leq\left\|D^{2} \gamma\right\|_{L^{\infty}\left(\bar{B}_{1}(0) \backslash B_{\alpha}(0)\right)} \int_{\Omega_{h}}\left|\bar{\nu}-\nu_{h}\right|\left|\nabla\left(\bar{u}_{t}-I_{h} \bar{u}_{t}\right)\right| \leq \int_{\Omega_{h}}\left|\bar{\nu}-\nu_{h}\right|^{2}+C h^{2}\left\|\bar{u}_{t}\right\|_{H^{2}\left(\Omega_{h}\right)}^{2},
$$

while

$$
\begin{aligned}
\left|I_{3}\right| & \leq\left\|\bar{u}_{t}\right\|_{L^{\infty}\left(\Omega_{h}\right)} \int_{\Omega_{h}}\left|\bar{\nu}-\nu_{h}\right|\left(\left|\bar{u}_{t}-I_{h} \bar{u}_{t}\right|+\left|\bar{u}_{t}-u_{h, t}\right|\right) \\
& \leq \varepsilon \int_{\Omega_{h}} \frac{\left|\bar{u}_{t}-u_{h, t}\right|^{2}}{\sqrt{1+\left|\nabla u_{h}\right|^{2}}}+C_{\varepsilon} \int_{\Omega_{h}}\left|\bar{\nu}-\nu_{h}\right|^{2} \sqrt{1+\left|\nabla u_{h}\right|^{2}}+C h^{2}\left\|\bar{u}_{t}\right\|_{H^{1}\left(\Omega_{h}\right)}^{2} .
\end{aligned}
$$

Finally,

$$
\left|I_{4}\right| \leq C\left(\left\|\bar{u}_{t}\right\|_{L^{\infty}\left(\Omega_{h}\right)}+\|\bar{u}\|_{H^{2, \infty}\left(\Omega_{h}\right)}\right)\left(\int_{\Omega_{h} \backslash \Omega}\left|\bar{u}_{t}-u_{h, t}\right|+\left|\Omega_{h} \backslash \Omega\right|^{\frac{1}{2}}\left\|\bar{u}_{t}-I_{h} \bar{u}_{t}\right\|_{L^{2}\left(\Omega_{h}\right)}\right) .
$$


The first integral can be estimated as follows:

$$
\begin{aligned}
\int_{\Omega_{h} \backslash \Omega}\left|\bar{u}_{t}-u_{h, t}\right| \leq & \int_{\Omega_{h} \backslash \Omega} \frac{\left|\bar{u}_{t}-u_{h, t}\right|}{\sqrt{1+\left|\nabla u_{h}\right|^{\frac{1}{2}}}} \sqrt{1+|\nabla \bar{u}|^{2}} \frac{1}{\frac{1}{2}} \\
& +\int_{\Omega_{h} \backslash \Omega} \frac{\left|\bar{u}_{t}-u_{h, t}\right|}{\sqrt{1+\left|\nabla u_{h}\right|^{\frac{1}{2}}}}\left|\sqrt{1+\left|\nabla u_{h}\right|^{\frac{1}{2}}}-\sqrt{1+|\nabla \bar{u}|^{2}}\right| \\
\leq & C \int_{\Omega_{h} \backslash \Omega} \frac{\left|\bar{u}_{t}-u_{h, t}\right|}{\sqrt{1+\left|\nabla u_{h}\right|^{2}}}+\int_{\Omega_{h} \backslash \Omega} \frac{\left|\bar{u}_{t}-u_{h, t}\right|}{{\sqrt{1+\left|\nabla u_{h}\right|^{2}}}^{\frac{1}{2}}} \frac{\left|\nabla\left(\bar{u}-u_{h}\right)\right|}{\sqrt{1+\left|\nabla u_{h}\right|^{2}}} .
\end{aligned}
$$

Combining Young's inequality with the estimate $\left|\Omega_{h} \backslash \Omega\right| \leq C h^{2}$ we get

$$
\left|I_{4}\right| \leq \epsilon \int_{\Omega_{h}} \frac{\left|\bar{u}_{t}-u_{h, t}\right|^{2}}{\sqrt{1+\left|\nabla u_{h}\right|^{2}}}+C_{\epsilon}\left(h^{2}+\int_{\Omega_{h}} \frac{\left|\nabla\left(\bar{u}-u_{h}\right)\right|^{2}}{\sqrt{1+\left|\nabla u_{h}\right|^{2}}}\right) .
$$

Since

$$
\frac{\left(\nabla\left(\bar{u}-u_{h}\right), 0\right)}{\sqrt{1+\left|\nabla u_{h}\right|^{2}}}=\bar{\nu}-\nu_{h}+\left(\bar{\nu}_{n+1}-\nu_{h, n+1}\right)(\nabla \bar{u},-1)
$$

we may estimate

$$
\left|\nabla\left(\bar{u}-u_{h}\right)\right| \leq C(M)\left|\bar{\nu}-\nu_{h}\right| \sqrt{1+\left|\nabla u_{h}\right|^{2}} .
$$

Inserting the above estimates into (3.5) and choosing $\varepsilon$ small we get

$$
\begin{aligned}
\frac{1}{2} \int_{\Omega_{h}} \frac{\left(\bar{u}_{t}-u_{h, t}\right)^{2}}{\sqrt{1+\left|\nabla u_{h}\right|^{2}}}+\sum_{i=1}^{n} \int_{\Omega_{h}}\left(\gamma_{p_{i}}(\bar{\nu})-\gamma_{p_{i}}\left(\nu_{h}\right)\right)\left(\bar{u}_{t, x_{i}}-u_{h t, x_{i}}\right) & \\
& \leq C\left(1+\left\|\bar{u}_{t}\right\|_{H^{2}\left(\Omega_{h}\right)}^{2}\right) h^{2}+C \int_{\Omega_{h}}\left|\bar{\nu}-\nu_{h}\right|^{2} \sqrt{1+\left|\nabla u_{h}\right|^{2}} .
\end{aligned}
$$

It remains to examine the second integral on the left-hand side above. Since $\gamma_{p_{i}}$ is homogeneous of degree zero and $\gamma_{p_{i} p_{j}}$ is homogeneous of degree -1 we have

$$
\begin{gathered}
\gamma_{p_{i}}(\bar{\nu})=\gamma_{p_{i}}(\nabla \bar{u},-1), \quad \gamma_{p_{i}}\left(\nu_{h}\right)=\gamma_{p_{i}}\left(\nabla u_{h},-1\right), \\
\gamma_{p_{i} p_{j}}(\bar{\nu})=\sqrt{1+|\nabla \bar{u}|^{2}} \gamma_{p_{i} p_{j}}(\nabla \bar{u},-1),
\end{gathered}
$$

and therefore

$$
\begin{aligned}
\sum_{i=1}^{n}\left(\gamma_{p_{i}}(\bar{\nu})-\gamma_{p_{i}}\left(\nu_{h}\right)\right)\left(\bar{u}_{t, x_{i}}-u_{h t, x_{i}}\right)= & \frac{\mathrm{d}}{\mathrm{d} t}\left[\gamma\left(\nabla u_{h},-1\right)-\sum_{i=1}^{n} \gamma_{p_{i}}(\nabla \bar{u},-1) u_{h, x_{i}}\right] \\
& +\sum_{i=1}^{n}\left(\gamma_{p_{i}}(\bar{\nu})-\gamma_{p_{i}}\left(\nu_{h}\right)\right) \bar{u}_{t, x_{i}}+\sum_{i, j=1}^{n} \gamma_{p_{i} p_{j}}(\nabla \bar{u},-1) u_{h, x_{j}} \bar{u}_{t, x_{i}} .
\end{aligned}
$$


Clearly,

$$
\begin{aligned}
\sum_{i=1}^{n}\left(\gamma_{p_{i}}(\bar{\nu})-\gamma_{p_{i}}\left(\nu_{h}\right)\right) \bar{u}_{t, x_{i}}= & \sum_{i=1}^{n} \sum_{j=1}^{n+1} \int_{0}^{1} \gamma_{p_{i} p_{j}}\left(s \bar{\nu}+(1-s) \nu_{h}\right) \mathrm{d} s\left(\bar{\nu}_{j}-\nu_{h, j}\right) \bar{u}_{t, x_{i}} \\
\sum_{i, j=1}^{n} \gamma_{p_{i} p_{j}}(\nabla \bar{u},-1) u_{h, x_{j}} \bar{u}_{t, x_{i}}= & \sum_{i, j=1}^{n} \gamma_{p_{i} p_{j}}(\bar{\nu}) \frac{u_{h, x_{j}}}{\sqrt{1+|\nabla \bar{u}|^{2}}} \bar{u}_{t, x_{i}} \\
= & \sum_{i, j=1}^{n} \gamma_{p_{i} p_{j}}(\bar{\nu}) \bar{\nu}_{j} \bar{u}_{t, x_{i}}-\sum_{i, j=1}^{n} \gamma_{p_{i} p_{j}}(\bar{\nu})\left(\bar{\nu}_{j}-\nu_{h, j}\right) \bar{u}_{t, x_{i}} \\
& +\sum_{i, j=1}^{n} \gamma_{p_{i} p_{j}}(\bar{\nu}) u_{h, x_{j}}\left(\frac{1}{\sqrt{1+|\nabla \bar{u}|^{2}}}-\frac{1}{\sqrt{1+\left|\nabla u_{h}\right|^{2}}}\right) \bar{u}_{t, x_{i}} .
\end{aligned}
$$

With $\mu:=\left(\nabla \bar{u}_{t}, 0\right) \in \mathbb{R}^{n+1}$ we conclude from $(1.4)$

$$
0=\sum_{i, j,=1}^{n+1} \gamma_{p_{i} p_{j}}(\bar{\nu}) \bar{\nu}_{j} \mu_{i}=\sum_{i, j=1}^{n} \gamma_{p_{i} p_{j}}(\bar{\nu}) \bar{\nu}_{j} \bar{u}_{t, x_{i}}+\sum_{i=1}^{n} \gamma_{p_{i} p_{n+1}}(\bar{\nu}) \bar{\nu}_{n+1} \bar{u}_{t, x_{i}}
$$

Observing that $\bar{\nu}_{n+1}=-1 / \sqrt{1+|\nabla \bar{u}|^{2}}$ we get

$$
\begin{aligned}
\sum_{i, j=1}^{n} \gamma_{p_{i} p_{j}}(\nabla \bar{u},-1) u_{h, x_{j}} \bar{u}_{t, x_{i}}= & \sum_{i=1}^{n} \gamma_{p_{i} p_{n+1}}(\nabla \bar{u},-1) \bar{u}_{t, x_{i}}-\sum_{i, j=1}^{n} \gamma_{p_{i} p_{j}}(\bar{\nu})\left(\bar{\nu}_{j}-\nu_{h, j}\right) \bar{u}_{t, x_{i}} \\
& -\left(\bar{\nu}_{n+1}-\nu_{h, n+1}\right) \sum_{i, j=1}^{n} \gamma_{p_{i} p_{j}}(\bar{\nu}) \bar{u}_{x_{j}} \bar{u}_{t, x_{i}} \\
& +\left(\bar{\nu}_{n+1}-\nu_{h, n+1}\right) \sum_{i, j=1}^{n} \gamma_{p_{i} p_{j}}(\bar{\nu})\left(\bar{u}_{x_{j}}-u_{h, x_{j}}\right) \bar{u}_{t, x_{i}} \\
= & \frac{\mathrm{d}}{\mathrm{d} t} \gamma_{p_{n+1}}(\nabla \bar{u},-1)-\sum_{i=1}^{n} \sum_{j=1}^{n+1} \gamma_{p_{i} p_{j}}(\bar{\nu})\left(\bar{\nu}_{j}-\nu_{h, j}\right) \bar{u}_{t, x_{i}} \\
& +\left(\bar{\nu}_{n+1}-\nu_{h, n+1}\right) \sum_{i, j=1}^{n} \gamma_{p_{i} p_{j}}(\bar{\nu})\left(\bar{u}_{x_{j}}-u_{h, x_{j}}\right) \bar{u}_{t, x_{i}}
\end{aligned}
$$

again by (3.10). Taking into account the above calculations we obtain from (3.9)

$$
\begin{aligned}
\sum_{i=1}^{n}\left(\gamma_{p_{i}}(\bar{\nu})-\gamma_{p_{i}}\left(\nu_{h}\right)\right)\left(\bar{u}_{t, x_{i}}-u_{h t, x_{i}}\right)= & \frac{\mathrm{d}}{\mathrm{d} t} E(t)+\sum_{i=1}^{n} \sum_{j=1}^{n+1} \int_{0}^{1}\left(\gamma_{p_{i} p_{j}}\left(s \bar{\nu}+(1-s) \nu_{h}\right)-\gamma_{p_{i} p_{j}}(\bar{\nu})\right)\left(\bar{\nu}_{j}-\nu_{h, j}\right) \bar{u}_{t, x_{i}} \\
& +\left(\bar{\nu}_{n+1}-\nu_{h, n+1}\right) \sum_{i, j=1}^{n} \gamma_{p_{i} p_{j}}(\bar{\nu})\left(\bar{u}_{x_{j}}-u_{h, x_{j}}\right) \bar{u}_{t, x_{i}} .
\end{aligned}
$$

A similar calculation as in (3.6) shows

$$
\left|\tau s \bar{\nu}+\tau(1-s) \nu_{h}+(1-\tau) \bar{\nu}\right| \geq \alpha, s, \tau \in[0,1]
$$


so that the lemma follows after inserting $(3.7,3.11)$ into (3.8).

The next step consists in estimating $E(t)$ from below.

Lemma 3.2. There exists a constant $c$, depending on $\gamma_{0}$ and an upper bound $M$ for $\|\nabla \bar{u}\|_{L^{\infty}\left(\bar{\Omega}^{\prime} \times[0, T]\right)}$, such that

$$
E(t) \geq c \int_{\Omega_{h}}\left|\bar{\nu}(\cdot, t)-\nu_{h}(\cdot, t)\right|^{2} \sqrt{1+\left|\nabla u_{h}(\cdot, t)\right|^{2}} .
$$

Proof. From (1.3) 1 and (1.4) we infer

$$
\begin{aligned}
E & =\int_{\Omega_{h}}\left(\gamma\left(\nu_{h}\right)-\left\langle\nabla \gamma(\bar{\nu}), \nu_{h}\right\rangle\right) \sqrt{1+\left|\nabla u_{h}\right|^{2}} \\
& =\int_{\Omega_{h}}\left(\gamma\left(\nu_{h}\right)-\gamma(\bar{\nu})-\left\langle\nabla \gamma(\bar{\nu}), \nu_{h}-\bar{\nu}\right\rangle\right) \sqrt{1+\left|\nabla u_{h}\right|^{2}} \\
& =\int_{\Omega_{h}} \int_{0}^{1}\left\langle\nabla \gamma\left(s \nu_{h}+(1-s) \bar{\nu}\right)-\nabla \gamma(\bar{\nu}), \nu_{h}-\bar{\nu}\right\rangle \mathrm{d} s \sqrt{1+\left|\nabla u_{h}\right|^{2}} \\
& =\int_{\Omega_{h}} \int_{0}^{1} s \int_{0}^{1}\left\langle D^{2} \gamma\left(s \tau \nu_{h}+(1-s \tau) \bar{\nu}\right)\left(\nu_{h}-\bar{\nu}\right), \nu_{h}-\bar{\nu}\right\rangle \mathrm{d} \tau \mathrm{d} s \sqrt{1+\left|\nabla u_{h}\right|^{2}} .
\end{aligned}
$$

Let us fix $s, \tau \in[0,1]$ and define $\mu_{s \tau}:=s \tau \nu_{h}+(1-s \tau) \bar{\nu}$. We decompose

$$
\nu_{h}-\bar{\nu}=\lambda \frac{\mu_{s \tau}}{\left|\mu_{s \tau}\right|}+\eta \text { with } \lambda \in \mathbb{R} \text { and }\left\langle\eta, \mu_{s \tau}\right\rangle=0
$$

Using the homogeneity of $D^{2} \gamma$ together with (1.3) and $(1.4)_{2}$ we get

$$
\left\langle D^{2} \gamma\left(\mu_{s \tau}\right)\left(\nu_{h}-\bar{\nu}\right), \nu_{h}-\bar{\nu}\right\rangle=\left\langle D^{2} \gamma\left(\frac{\mu_{s \tau}}{\left|\mu_{s \tau}\right|}\right) \eta, \eta\right\rangle \frac{1}{\left|\mu_{s \tau}\right|} \geq \gamma_{0}|\eta|^{2} .
$$

On the other hand

$$
\lambda=\frac{1}{\left|\mu_{s \tau}\right|}\left\langle\nu_{h}-\bar{\nu}, \bar{\nu}+s \tau\left(\nu_{h}-\bar{\nu}\right)\right\rangle=\frac{1}{\left|\mu_{s \tau}\right|}\left(\left\langle\nu_{h}, \bar{\nu}\right\rangle-1+s \tau\left|\nu_{h}-\bar{\nu}\right|^{2}\right) .
$$

Since $\left|\nu_{h}-\bar{\nu}\right|^{2}=2-2\left\langle\nu_{h}, \bar{\nu}\right\rangle$ we deduce

$$
\begin{aligned}
\lambda & =\frac{1}{\left|\mu_{s \tau}\right|}\left(s \tau-\frac{1}{2}\right)\left|\nu_{h}-\bar{\nu}\right|^{2} \\
\left|\mu_{s \tau}\right|^{2} & =1+s \tau(s \tau-1)\left|\nu_{h}-\bar{\nu}\right|^{2} .
\end{aligned}
$$

Thus,

$$
\lambda^{2}=\frac{\left(s \tau-\frac{1}{2}\right)^{2}\left|\nu_{h}-\bar{\nu}\right|^{2}}{1+s \tau(s \tau-1)\left|\nu_{h}-\bar{\nu}\right|^{2}} \cdot\left|\nu_{h}-\bar{\nu}\right|^{2} .
$$


Taking into account that

$$
\begin{aligned}
\left|\nu_{h}-\bar{\nu}\right|^{2} & =2-2 \frac{\nabla \bar{u} \cdot \nabla u_{h}+1}{\sqrt{1+|\nabla \bar{u}|^{2}} \sqrt{1+\left|\nabla u_{h}\right|^{2}}} \leq 2+2 \frac{|\nabla \bar{u}|}{\sqrt{1+|\nabla \bar{u}|^{2}}} \\
& \leq 2+2 \frac{M}{\sqrt{1+M^{2}}}<4
\end{aligned}
$$

we see that

$$
\frac{\left(s \tau-\frac{1}{2}\right)^{2}\left|\nu_{h}-\bar{\nu}\right|^{2}}{1+s \tau(s \tau-1)\left|\nu_{h}-\bar{\nu}\right|^{2}}=\frac{\left(s \tau-\frac{1}{2}\right)^{2}\left|\nu_{h}-\bar{\nu}\right|^{2}}{\left(s \tau-\frac{1}{2}\right)^{2}\left|\nu_{h}-\bar{\nu}\right|^{2}+1-\frac{1}{4}\left|\nu_{h}-\bar{\nu}\right|^{2}} \leq \rho<1
$$

uniformly in $s, \tau \in[0,1]$. Thus

$$
\left|\nu_{h}-\bar{\nu}\right|^{2}=\lambda^{2}+|\eta|^{2} \leq \rho\left|\nu_{h}-\bar{\nu}\right|^{2}+|\eta|^{2}
$$

or equivalently

$$
(1-\rho)\left|\nu_{h}-\bar{\nu}\right|^{2} \leq|\eta|^{2}
$$

Returning to (3.12) we infer

$$
\left\langle D^{2} \gamma\left(\mu_{s \tau}\right)\left(\nu_{h}-\bar{\nu}\right), \nu_{h}-\bar{\nu}\right\rangle \geq(1-\rho) \gamma_{0}\left|\nu_{h}-\bar{\nu}\right|^{2} \forall s, \tau \in[0,1]
$$

so that

$$
E \geq \frac{1}{2}(1-\rho) \gamma_{0} \int_{\Omega_{h}}\left|\nu_{h}-\bar{\nu}\right|^{2} \sqrt{1+\left|\nabla u_{h}\right|^{2}}
$$

Now we are in position to prove Theorem 2.1. From Lemma 3.1 we obtain after integration with respect to $s \in(0, t)$

$$
\frac{1}{2} \int_{0}^{t} \int_{\Omega_{h}} \frac{\left|\bar{u}_{t}-u_{h, t}\right|^{2}}{\sqrt{1+\left|\nabla u_{h}\right|^{2}}}+E(t) \leq E(0)+C h^{2}+C \int_{0}^{t} \int_{\Omega_{h}}\left|\bar{\nu}-\nu_{h}\right|^{2} \sqrt{1+\left|\nabla u_{h}\right|^{2}} .
$$

It is easy to see that $E(0) \leq C h^{2}$ so that Lemma 3.2 and (3.11) yield

$$
\int_{\Omega_{h}}\left|\left(\bar{\nu}-\nu_{h}\right)(\cdot, t)\right|^{2} \sqrt{1+\left|\nabla u_{h}(\cdot, t)\right|^{2}} \leq C h^{2}+C \int_{0}^{t} \int_{\Omega_{h}}\left|\bar{\nu}-\nu_{h}\right|^{2} \sqrt{1+\left|\nabla u_{h}\right|^{2}} .
$$

Gronwall's lemma implies

$$
\sup _{t \in(0, T)} \int_{\Omega_{h}}\left|\left(\bar{\nu}-\nu_{h}\right)(\cdot, t)\right|^{2} \sqrt{1+\left|\nabla u_{h}(\cdot, t)\right|^{2}} \leq C h^{2}
$$


and then by (3.13)

$$
\int_{0}^{T} \int_{\Omega_{h}} \frac{\left|\bar{u}_{t}-u_{h, t}\right|^{2}}{\sqrt{1+\left|\nabla u_{h}\right|^{2}}} \leq C h^{2} .
$$

Combining this estimate with (3.14) and

$$
-\bar{V}+V_{h}=\frac{\bar{u}_{t}-u_{h, t}}{\sqrt{1+\left|\nabla u_{h}\right|^{2}}}+\left(\bar{\nu}_{n+1}-\nu_{h, n+1}\right) \bar{u}_{t}
$$

finally gives

$$
\int_{0}^{T} \int_{\Omega_{h}}\left|\bar{V}-V_{h}\right|^{2} \sqrt{1+\left|\nabla u_{h}\right|^{2}} \leq C h^{2} .
$$

As $\bar{u}=u$ on $\left(\Omega_{h} \cap \Omega\right) \times(0, T)$ the proof is complete.

Remark 3.3. (i) Note that our convergence proof only requires $\gamma$ to be positively homogeneous of degree one, i.e. $\gamma(\lambda p)=\lambda \gamma(p)$ for all $\lambda>0$. The stronger condition $(1.3)_{1}$ is only needed in the proof of the global existence theorem $(c f .(4.7))$.

(ii) In the important case $n=2$ Theorem 2.1 directly implies the following error estimates for the function $u$ itself:

$$
\int_{0}^{T}\left\|u_{t}-u_{h, t}\right\|_{L^{2}\left(\Omega_{h} \cap \Omega\right)}^{2} \mathrm{~d} t+\sup _{t \in(0, T)}\left\|\nabla\left(u-u_{h}\right)(\cdot, t)\right\|_{L^{2}\left(\Omega_{h} \cap \Omega\right)}^{2} \leq C h^{2} .
$$

To see this, we use (3.11) and Theorem 2.1 to estimate

$$
\int_{\Omega_{h}} \frac{\left|\nabla\left(\bar{u}-u_{h}\right)\right|^{2}}{\sqrt{1+\left|\nabla u_{h}\right|^{2}}} \leq C \int_{\Omega_{h}}\left|\bar{\nu}-\nu_{h}\right|^{2} \sqrt{1+\left|\nabla u_{h}\right|^{2}} \leq C h^{2} .
$$

Just as in the proof of Lemma 2 in [9] this implies

$$
\sup _{t \in(0, T)} \sup _{\Omega_{h}}\left|\nabla u_{h}(\cdot, t)\right| \leq C
$$

and the above bound follows.

\section{Existence of A SOLUtion}

In the following section we give an existence and uniqueness result for (1.2) in function spaces which are adequate for our error analysis.

Theorem 4.1. Let $\partial \Omega \in C^{3+\alpha}$ and $d(x):=\operatorname{dist}(x, \partial \Omega)$. We assume that $\gamma$ satisfies (1.3) as well as

$$
\sum_{i, j=1}^{n} \gamma_{p_{i} p_{j}}(\nabla d(x), 0) d_{x_{i} x_{j}}(x) \leq 0, x \in \partial \Omega .
$$


Let $u_{0} \in C^{3+\alpha}(\bar{\Omega})$ satisfy the compatibility condition

$$
\sum_{i, j=1}^{n} \gamma_{p_{i} p_{j}}\left(\nabla u_{0}(x),-1\right) u_{0, x_{i} x_{j}}(x)=0, x \in \partial \Omega
$$

Then (1.2) has a unique solution $u \in H^{3+\alpha, \frac{3+\alpha}{2}}(\bar{\Omega} \times[0, T])$ with $u_{t} \in L^{2}\left(0, T ; H^{2,2}(\Omega)\right)$ for all $T<\infty$.

The definition of the parabolic Hölder spaces $H^{k+\alpha, \frac{k+\alpha}{2}}(\bar{\Omega} \times[0, T]),(k \in \mathbb{N}, 0<\alpha<1)$ can be found in [10]. If (4.1) is not satisfied a solution with the above regularity exists in general only locally and singularities may develop in finite time ( $c f .[12])$.

Proof of Theorem 4.1 We are looking for a solution of the initial boundary value problem

$$
\begin{aligned}
u_{t}-\sum_{i, j=1}^{n} a_{i j}(\nabla u) u_{x_{i} x_{j}} & =0 & & \text { in } \Omega \times(0, T) \\
u & =u_{0} & & \text { on } \partial \Omega \times(0, T) \\
u(\cdot, 0) & =u_{0} & & \text { in } \Omega
\end{aligned}
$$

where

$$
a_{i j}(\tilde{p}):=\sqrt{1+|\tilde{p}|^{2}} \gamma_{p_{i} p_{j}}(\tilde{p},-1), \tilde{p} \in \mathbb{R}^{n} .
$$

We first show that $\left(a_{i j}(\tilde{p})\right)_{i, j=1}^{n}$ is elliptic. To this purpose, let $\xi \in \mathbb{R}^{n}, \hat{\xi}=(\xi, 0)^{t} \in \mathbb{R}^{n+1}$ and write

$$
\hat{\xi}=\lambda \frac{1}{\sqrt{1+|\tilde{p}|^{2}}}(\tilde{p},-1)^{t}+\eta,\left\langle\eta,(\tilde{p},-1)^{t}\right\rangle=0 .
$$

The homogeneity of $D^{2} \gamma$ together with $(1.4)_{2}$ and $(1.3)_{2}$ gives

$$
\sum_{i, j=1}^{n} a_{i j}(\tilde{p}) \xi_{i} \xi_{j}=\sum_{i, j=1}^{n+1} \gamma_{p_{i} p_{j}}\left(\frac{(\tilde{p},-1)^{t}}{\sqrt{1+|\tilde{p}|^{2}}}\right) \hat{\xi}_{i} \hat{\xi}_{j}=\left\langle D^{2} \gamma\left(\frac{(\tilde{p},-1)^{t}}{\sqrt{1+|\tilde{p}|^{2}}}\right) \eta, \eta\right\rangle \geq \gamma_{0}|\eta|^{2} .
$$

Since $|\lambda| \leq \frac{|\tilde{p}||\xi|}{\sqrt{1+|\tilde{p}|^{2}}}$ we get

$$
|\xi|^{2}=|\hat{\xi}|^{2}=\lambda^{2}+|\eta|^{2} \leq \frac{|\tilde{p}|^{2}}{1+|\tilde{p}|^{2}}|\xi|^{2}+|\eta|^{2}
$$

which, substituted into (4.3) implies

$$
\sum_{i, j=1}^{n} a_{i j}(\tilde{p}) \xi_{i} \xi_{j} \geq \frac{\gamma_{0}}{1+|\tilde{p}|^{2}}|\xi|^{2}
$$

Furthermore, we conclude that the largest eigenvalue $\Lambda(\tilde{p})$ and the smallest eigenvalue $\lambda(\tilde{p})$ of $\left(a_{i j}(\tilde{p})\right)_{i, j}$ satisfy

$$
\Lambda(\tilde{p}) \leq \Lambda_{0}, \lambda(\tilde{p}) \geq \frac{\gamma_{0}}{1+|\tilde{p}|^{2}} .
$$


We employ the results of [11] in order to prove existence of a solution of (4.2). In view of Lemma 5.1 and Corollary 5.2 in [11] (4.2) has a solution $u \in H^{2+\alpha, 1+\frac{\alpha}{2}}(\bar{\Omega} \times[0, T])$ provided there is a constant $K$ such that for every $\tau \in[0,1]$ and every solution $u^{\tau}$ of

$$
\begin{array}{rlll}
u_{t}^{\tau}-\tau \sum_{i, j=1}^{n} a_{i j}\left(\nabla u^{\tau}\right) u_{x_{i} x_{j}}^{\tau}-(1-\tau) \Delta u^{\tau} & =0 & & \text { in } \Omega \times(0, T) \\
u^{\tau} & =\tau u_{0} & & \text { on } \partial \Omega \times(0, T) \\
u^{\tau}(\cdot, 0) & =\tau u_{0} & & \text { on } \Omega
\end{array}
$$

the estimate

$$
\sup _{\Omega \times(0, T)}\left|u^{\tau}\right|+\sup _{\Omega \times(0, T)}\left|\nabla u^{\tau}\right| \leq K
$$

is valid. To begin, the parabolic maximum principle implies

$$
\sup _{\Omega \times(0, T)}\left|u^{\tau}\right| \leq \sup _{\Omega}\left|u_{0}\right| .
$$

In order to obtain a boundary gradient estimate we apply Theorem 2.6 in [11]. Let us decompose the coefficients $a_{i j}(\tau, \tilde{p})=\tau a_{i j}(\tilde{p})+(1-\tau) \delta_{i j}$ for $\tilde{p} \neq 0$ as follows:

$$
a_{i j}(\tau, \tilde{p})=a_{i j}^{\infty}\left(\frac{\tilde{p}}{|\tilde{p}|}\right) \Lambda(\tilde{p})+a_{i j}^{0}(\tau, \tilde{p}) i, j=1, \ldots, n
$$

where

$$
\begin{array}{rlrl}
a_{i j}^{\infty}(\zeta) & =\tilde{\Lambda}^{-1}(\zeta) \gamma_{p_{i} p_{j}}(\zeta, 0), & & \zeta \in S^{n-1} \\
\tilde{\Lambda}(\zeta) & =\sup _{|\eta|=1} \sum_{i, j=1}^{n} \gamma_{p_{i} p_{j}}(\zeta, 0) \eta_{i} \eta_{j}, & \zeta \in S^{n-1} \\
a_{i j}^{0}(\tau, \tilde{p}) & =a_{i j}(\tau, \tilde{p})-a_{i j}^{\infty}\left(\frac{\tilde{p}}{|\tilde{p}|}\right) \Lambda(\tilde{p}) . &
\end{array}
$$

Similarly as above, we deduce that $a_{i j}^{\infty}(\zeta)$ is positive semidefinite and it is not hard to show that $a_{i j}^{\infty}$ is a Lipschitz function on $S^{n-1}$. Furthermore, (4.4) implies

$$
\tau \Lambda(\tilde{p})+(1-\tau) \leq \mu_{1}(\tau \lambda(\tilde{p})+(1-\tau))|p|^{2} \text { for }|p| \geq 1, \mu_{1}=\max \left(1, \frac{2 \Lambda_{0}}{\gamma_{0}}\right) .
$$

Since $u_{0}$ is independent of time we see that $(2.6,2.13)$ in [11] are satisfied. Next, $(1.4)_{2}$ and (4.1) yield

$$
-\sup _{y \in \partial \Omega} \sum_{i, j=1}^{n} a_{i j}^{\infty}( \pm \nabla d(y)) d_{x_{i} x_{j}}(y)=-\sup _{y \in \partial \Omega} \frac{1}{\tilde{\Lambda}(\nabla d(y))} \sum_{i, j=1}^{n} \gamma_{p_{i} p_{j}}( \pm \nabla d(y), 0) d_{x_{i} x_{j}}(y) \geq 0
$$

which is the first condition in $(2.20 \mathrm{~b})$ in [11]. In order to check the second one we have to control $\sigma(\tau, \tilde{p}):=$ $|\tilde{p}| \sup _{|\xi|=1} \sum_{i, j=1}^{n} a_{i j}^{0}(\tau, \tilde{p}) \xi_{i} \xi_{j}$. Note that

$$
\left|\gamma_{p_{i} p_{j}}\left(\frac{(\tilde{p},-1)}{\sqrt{1+|\tilde{p}|^{2}}}\right)-\frac{\Lambda(\tilde{p})}{\tilde{\Lambda}\left(\frac{\tilde{\tilde{p}}}{|\tilde{p}|}\right)} \gamma_{p_{i} p_{j}}\left(\frac{\tilde{p}}{|\tilde{p}|}, 0\right)\right| \leq C\left|\frac{(\tilde{p},-1)}{\sqrt{1+|\tilde{p}|^{2}}}-\left(\frac{\tilde{p}}{|\tilde{p}|}, 0\right)\right| \leq \frac{C}{|\tilde{p}|}
$$


since

$$
\left|s \frac{(\tilde{p},-1)}{\sqrt{1+|\tilde{p}|^{2}}}+(1-s)\left(\frac{\tilde{p}}{|\tilde{p}|}, 0\right)\right| \geq \frac{1}{\sqrt{2}}, s \in[0,1] .
$$

Thus,

$$
\sigma(\tau, \tilde{p}) \leq C \tau+|\tilde{p}|(1-\tau)
$$

while

$$
\begin{aligned}
\mathcal{E}(\tau, \tilde{p}):=\sum_{i, j=1}^{n} a_{i j}(\tau, \tilde{p}) \tilde{p}_{i} \tilde{p}_{j} & \geq \frac{\tau \gamma_{0}|\tilde{p}|^{2}}{1+|\tilde{p}|^{2}}+(1-\tau)|\tilde{p}|^{2} \\
& \geq \frac{1}{2} \tau \gamma_{0}+(1-\tau)|\tilde{p}| \text { for }|\tilde{p}| \geq 1 .
\end{aligned}
$$

Therefore, $\sigma(\tau, \tilde{p})=O(\mathcal{E}(\tau, \tilde{p}))$ as $|\tilde{p}| \rightarrow \infty$. We have thus verified all the conditions for Theorem 2.6 in [11] and conclude

$$
\sup _{\partial \Omega \times(0, T)}\left|\nabla u^{\tau}\right| \leq K_{1}
$$

Finally, the bound on $\sup _{\Omega \times(0, T)}\left|\nabla u^{\tau}\right|$ can be obtained by deriving for $v^{\tau}:=\sqrt{1+\left|\nabla u^{\tau}\right|^{2}}$ an evolution inequality of the form

$$
v_{t}^{\tau}-\tau \sum_{i, j=1}^{n} a_{i j}\left(\nabla u^{\tau}\right) u_{x_{i} x_{j}}^{\tau}-(1-\tau) \Delta v^{\tau}+\sum_{i=1}^{n} b_{i} v_{x_{i}}^{\tau} \leq 0
$$

and applying the maximum principle with (4.8) (see [8] for a similar argument). Thus, (4.6) is proved and as already mentioned above the existence of a solution $u \in H^{2+\alpha, 1+\frac{\alpha}{2}}(\bar{\Omega} \times[0, T])$ of (4.2) follows.

Note in particular that $\tilde{a}_{i j}(x, t)=a_{i j}(\nabla u(x, t)) \in H^{1+\alpha, \frac{1+\alpha}{2}}(\bar{\Omega} \times[0, T]), i, j=1, \ldots, n$. From our assumptions on $u_{0}, \partial \Omega$ and Theorem 5.2, Chapter IV in [10] we infer that $u \in H^{3+\alpha, \frac{3+\alpha}{2}}(\bar{\Omega} \times[0, T])$. It remains to prove that $u_{t} \in L^{2}\left(0, T ; H^{2,2}(\Omega)\right)$. A straightforward calculation shows

$$
\int_{0}^{T-\delta}\left\|\frac{u_{t}(\cdot, t+h)-u_{t}(\cdot, t)}{h}\right\|_{L^{2}(\Omega)}^{2} \mathrm{~d} t \leq C\left(\left\|\tilde{a}_{i j}\right\|_{H^{1+\alpha} \frac{1+\alpha}{2}},\|u\|_{H^{3+\alpha}, \frac{3+\alpha}{2}}, T\right)
$$

for all $h<\delta, \delta>0$. Since

$$
\sum_{i, j=1}^{n} \tilde{a}_{i j}(\cdot, t)\left[\frac{u_{x_{i} x_{j}}(\cdot, t+h)-u_{x_{i} x_{j}}(\cdot, t)}{h}\right]=\frac{u_{t}(\cdot, t+h)-u_{t}(\cdot, t)}{h}-\sum_{i, j=1}^{n} \frac{\tilde{a}_{i j}(\cdot, t+h)-\tilde{a}_{i j}(\cdot, t)}{h} u_{x_{i} x_{j}}(\cdot, t+h)
$$

for $0 \leq t \leq T-\delta$, elliptic regularity theory together with (4.9) gives

$$
\int_{0}^{T-\delta}\left\|\frac{D^{2} u(\cdot, t+h)-D^{2} u(\cdot, t)}{h}\right\|_{L^{2}(\Omega)}^{2} \leq C\left(\left\|\tilde{a}_{i j}\right\|_{H^{1+\alpha, \frac{1+\alpha}{2}}},\|u\|_{H^{3+\alpha, \frac{3+\alpha}{2}}}, T\right) .
$$

The result follows by sending $h \rightarrow 0, \delta \rightarrow 0$. 

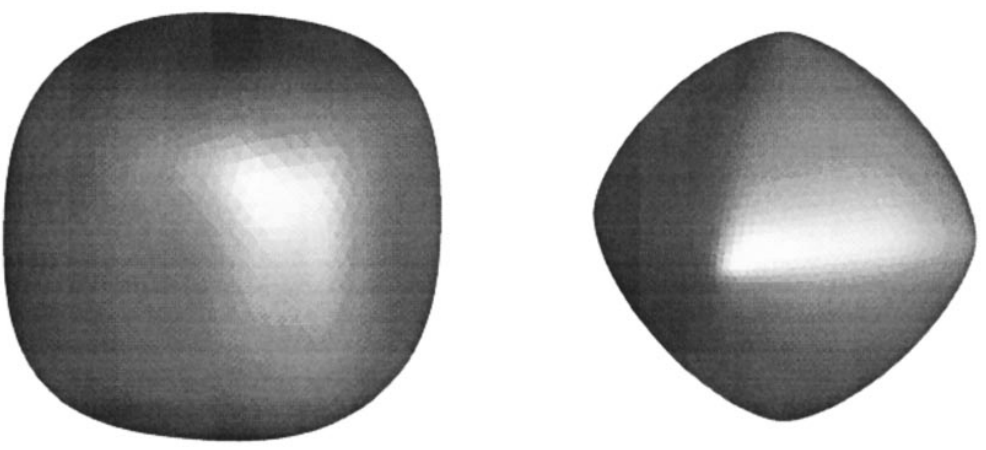

Figure 1. $\mathcal{F}$ and $\mathcal{W}$ for $A=0.24$.
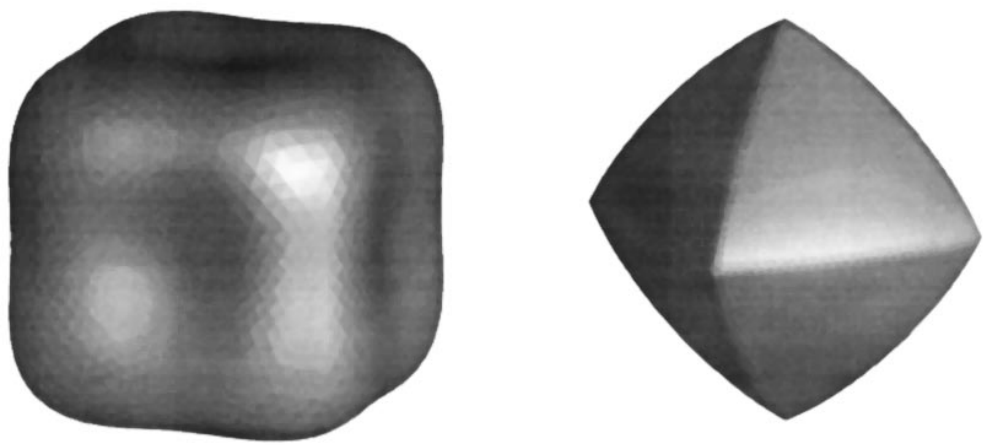

Figure 2. $\mathcal{F}$ and $\mathcal{W}$ for $A=0.5$ (scaled).

\section{NumERICAL RESULTS}

The nonlinear problem (2.3) is rewritten by using the relation

$$
\gamma(p)=\gamma_{0}(p)|p|
$$

and thus, using the 0-homogeneity of $\gamma_{0}$,

$$
\gamma_{p_{j}}(p)=\gamma_{0}(p) \frac{p_{j}}{|p|}+\gamma_{0 p_{j}}(p)|p|=\gamma\left(\frac{p}{|p|}\right) \frac{p_{j}}{|p|}+\gamma_{0 p_{j}}\left(\frac{p}{|p|}\right) .
$$

The equivalent nonlinear equation

$$
\int_{\Omega_{h}} \frac{u_{h, t} \varphi_{h}}{\sqrt{1+\left|\nabla u_{h}\right|^{2}}}+\int_{\Omega_{h}} \gamma\left(\frac{\left(\nabla u_{h},-1\right)}{\sqrt{1+\left|\nabla u_{h}\right|^{2}}}\right) \frac{\nabla u_{h} \nabla \varphi_{h}}{\sqrt{1+\left|\nabla u_{h}\right|^{2}}}=-\int_{\Omega_{h}} \sum_{j=1}^{n} \gamma_{0 p_{j}}\left(\frac{\left(\nabla u_{h},-1\right)}{\sqrt{1+\left|\nabla u_{h}\right|^{2}}}\right) \varphi_{h x_{j}} \quad \forall \varphi_{h} \in \dot{X}_{h}
$$

is then used to formulate a time discretization which leads to a linear problem. It is convenient to use time discretization for linearization too. Let $\tau>0$ be the time step size and let us use the notation

$$
v^{m}(x)=v(x, m \tau) \quad\left(m=0, \ldots, N=\left[\frac{T}{\tau}\right]\right)
$$



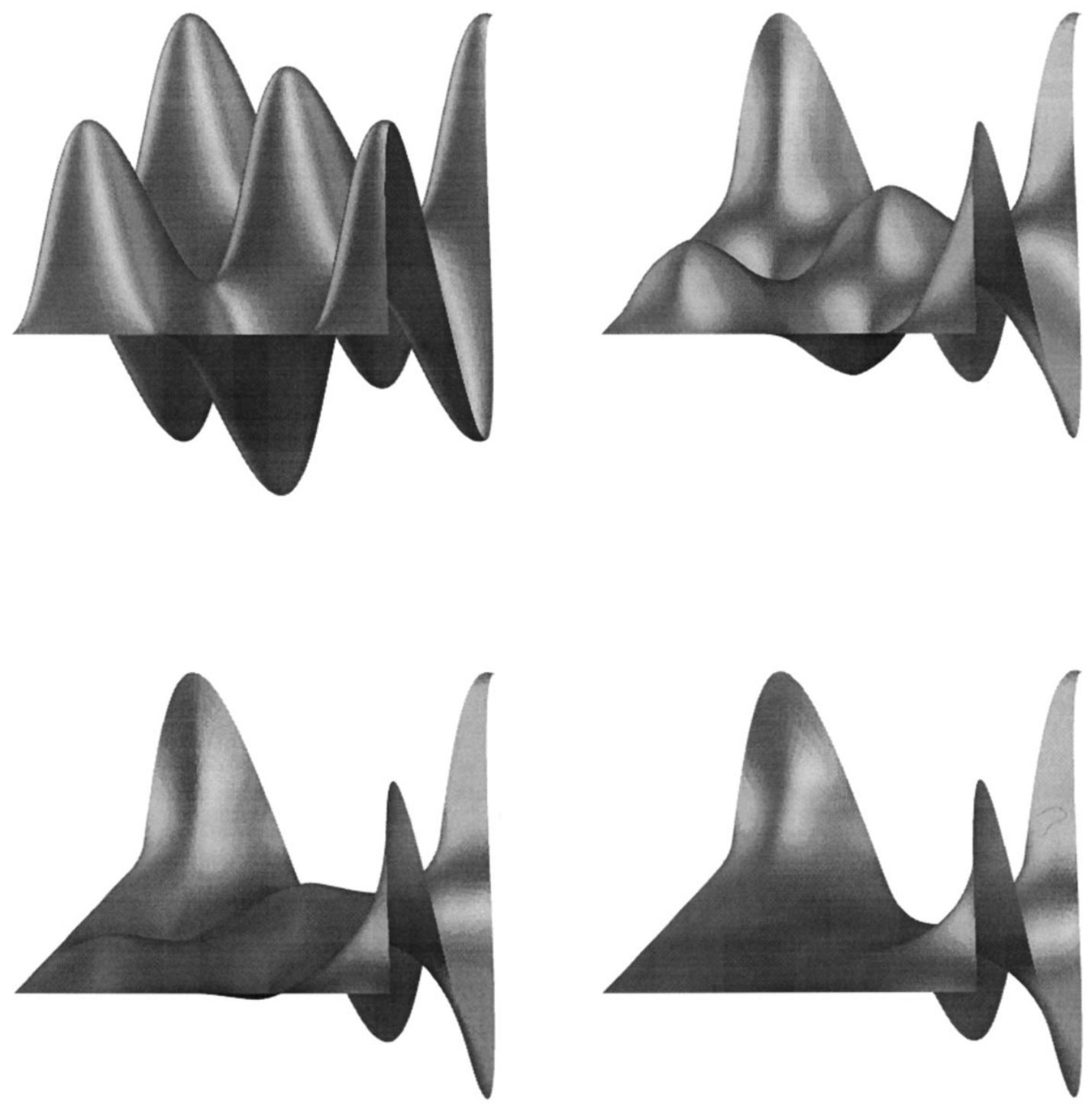

Figure 3. Evolution $(t=0.0, t=0.05, t=0.1, t=0.6)$ for $A=0.24$.

for a given function $v=v(x, t)$. The semidiscrete scheme is then discretised in the following way: find $u_{h}^{m} \in$ $X_{h}(m=1, \ldots, N)$ such that $u_{h}^{m}-I_{h} u_{0} \in \dot{X}_{h}$ and

$$
\begin{aligned}
\frac{1}{\tau} \int_{\Omega_{h}} \frac{u_{h}^{m} \varphi_{h}}{\sqrt{1+\left|\nabla u_{h}^{m-1}\right|^{2}}} & +\int_{\Omega_{h}} \gamma\left(\frac{\left(\nabla u_{h}^{m-1},-1\right)}{\sqrt{1+\left|\nabla u_{h}^{m-1}\right|^{2}}}\right) \frac{\nabla u_{h}^{m} \nabla \varphi_{h}}{\sqrt{1+\left|\nabla u_{h}^{m-1}\right|^{2}}} \\
& =\frac{1}{\tau} \int_{\Omega_{h}} \frac{u_{h}^{m-1} \varphi_{h}}{\sqrt{1+\left|\nabla u_{h}^{m-1}\right|^{2}}}-\int_{\Omega_{h}} \sum_{j=1}^{n} \gamma_{0 p_{j}}\left(\frac{\left(\nabla u_{h}^{m-1},-1\right)}{\sqrt{1+\left|\nabla u_{h}^{m-1}\right|^{2}}}\right) \varphi_{h x_{j}} \quad \forall \varphi_{h} \in \stackrel{X}{h}_{h} \\
u_{h}^{0} & =I_{h} u_{0} .
\end{aligned}
$$



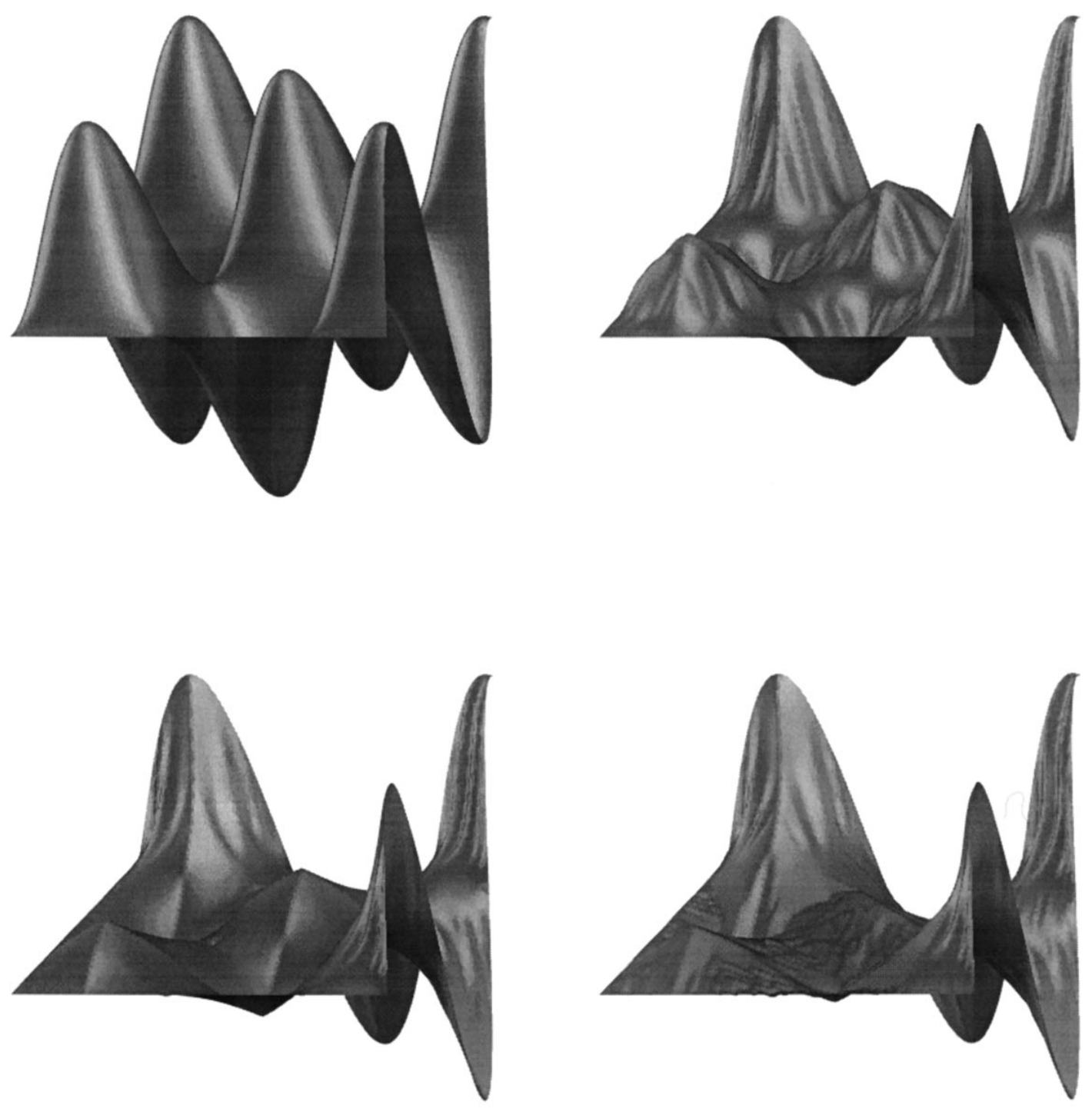

Figure 4. Evolution for $A=0.5$.

For better presentation let us assume that $\left.u_{0}\right|_{\partial \Omega}=0$ for the next lines. If we use the nodal basis $\dot{X}_{h}=$ $\operatorname{span}\left\{\varphi_{1}, \ldots, \varphi_{N}\right\}$, the resulting system of linear equations for the computation of $U^{m}=\left(U_{1}^{m}, \ldots, U_{N}^{m}\right)$,

$$
u_{h}^{m}(x)=\sum_{k=1}^{N} U_{k}^{m} \varphi_{k}(x), x \in \Omega_{h}
$$

within each time step is given by

$$
\left(\frac{1}{\tau} M^{(m-1)}+S^{(m-1)}\right) U^{m}=\frac{1}{\tau} M^{(m-1)} U^{m-1}-b^{(m-1)},
$$


where $M^{(m-1)}=\left(M^{(m-1)}\right)_{i, j=1, N}$,

$$
M_{i j}^{(m-1)}=\int_{\Omega_{h}} \frac{\varphi_{i} \varphi_{j}}{\sqrt{1+\left|\nabla u_{h}^{m-1}\right|^{2}}}
$$

is a weighted mass matrix, $S^{(m-1)}=\left(S^{(m-1)}\right)_{i, j=1, N}$,

$$
S_{i j}^{(m-1)}=\int_{\Omega_{h}} \gamma\left(\frac{\left(\nabla u_{h}^{m-1},-1\right)}{\sqrt{1+\left|\nabla u_{h}^{m-1}\right|^{2}}}\right) \frac{\nabla \varphi_{i} \nabla \varphi_{j}}{\sqrt{1+\left|\nabla u_{h}^{m-1}\right|^{2}}}
$$

is a weighted stiffness matrix and $b^{(m-1)}=\left(b^{(m-1)}\right)_{i=1, N}$,

$$
b_{i}^{(m-1)}=\int_{\Omega_{h}} \sum_{j=1}^{n} \gamma_{0 p_{j}}\left(\frac{\left(\nabla u_{h}^{m-1},-1\right)}{\sqrt{1+\left|\nabla u_{h}^{m-1}\right|^{2}}}\right) \varphi_{i x_{j}} .
$$

This shows that in each time step a linear system has to be solved numerically which is similar to that of a linear parabolic equation with variable coefficients. For bounded gradients $\nabla u_{h}^{m-1}$ the condition of (5.2) and as a consequence the computation time is also similar to that case. This fully discrete scheme has experimentally proved to be particularly stable.

We use the anisotropy function

$$
\gamma(p)=\left(1-A\left(1-\frac{|p|_{4}^{4}}{|p|^{4}}\right)\right)|p| \quad\left(p \in \mathbb{R}^{n+1} \backslash\{0\}\right)
$$

where

$$
|p|_{4}=\left(\sum_{j=1}^{n+1} p_{j}^{4}\right)^{\frac{1}{4}}
$$

and $0 \leq A \leq 1$ is a parameter. The amount of anisotropy imposed by (5.3) onto the problem can easily be visualised by plotting Frank diagram

$$
\mathcal{F}=\left\{p \in \mathbb{R}^{n+1} \mid \gamma(p) \leq 1\right\}
$$

and Wulff shape

$$
\mathcal{W}=\left\{q \in \mathbb{R}^{n+1} \mid \gamma^{*}(q) \leq 1\right\}
$$

using the dual $\gamma^{*}$ of $\gamma$ :

$$
\gamma^{*}(q)=\sup _{p \in \mathbb{R}^{n+1} \backslash\{0\}} \frac{\langle p, q\rangle}{\gamma(p)} .
$$

For $A=0$ which is the isotropic case

$$
\mathcal{F}=\mathcal{W}=\overline{B_{1}(0)}
$$

It is easy to check that for $A<0.25$ the convexity condition $(1.3)_{2}$ is satisfied, while for $A=0.5$ we do not have convexity. 
In the following we give two time series of anisotropic curvature flow on the domain $\Omega=(0,2) \times(0,2) \subset \mathbb{R}^{2}$ with initial condition

$$
u_{0}\left(x_{1}, x_{2}\right)=\sin \left(4 x_{1}\right) \sin \left(4 x_{2}\right) .
$$

The case $A=0.5$ is purely experimental although it is worth mentioning that the algorithm also seems to work for the nonconvex case. The grid contains 16641 nodes.

The pictures shown above were created using the Grape visualization package (see e.g. [13]).

\section{REFERENCES}

[1] S.B. Angenent and M.E. Gurtin, Multiphase thermomechanics with interfacial structure 2, evolution of an isothermal interface. Arch. Rational Mech. Anal. 108 (1989) 323-391.

[2] G. Bellettini and M. Paolini, Anisotropic motion by mean curvature in the context of Finsler geometry. Hokkaido Math. J. 25 (1996) 537-566.

[3] P. Clément, Approximation by finite element functions using local regularization. RAIRO Anal. Numér. 9 (1975) 77-84.

[4] K. Deckelnick and G. Dziuk, Convergence of a finite element method for non-parametric mean curvature flow. Numer. Math. 72 (1995) 197-222.

[5] M. Dobrowolski, $L^{\infty}$-convergence of linear finite element approximation to nonlinear parabolic problems. SIAM J. Numer. Anal. 17 (1980) 663-674.

[6] Y. Giga, Motion of a graph by convexified energy. Hokkaido Math. J. 23 (1994) 185-212.

[7] P.M. Girão and R.V. Kohn, Convergence of a crystalline algorithm for the heat equation in one dimension and for the motion of a graph by weighted curvature. Numer. Math. 67 (1994) 41-70.

[8] G. Huisken, Non-parametric mean curvature evolution with boundary conditions. J. Differential Equations 77 (1989) 369-378.

[9] C. Johnson and V. Thomeé, Error estimates for a finite element approximation of a minimal surface. Math. Comp. 29 (1975) 343-349.

[10] O.A. Ladyzhenskaya, V.A. Solonnikov and N.N. Ural'tseva, Linear and quasilinear equations of parabolic type. Amer. Math. Soc., Providence, R.I. (1968).

[11] G.A. Lieberman, The first initial-boundary value problem for quasilinear second order parabolic equations. Ann. Scuola Norm. Sup. Pisa Cl. Sci. Ser. IV 8 (1986) 347-387.

[12] V.I. Oliker and N.N. Uraltseva, Evolution of nonparametric surfaces with speed depending on curvature, II. The mean curvature case. Preprint.

[13] M. Rumpf, et al., GRAPE, Graphics Programming Environment. Report 8, SFB 256, Bonn (1990).

[14] J.E. Taylor, J.W. Cahn and C.A. Handwerker, Geometric models of crystal growth. Acta Metall. Mater. $401443-1474$ (1992). 